\title{
Clinical characteristics and outcome of hydrocephalus in neurosarcoidosis: a retrospective cohort study and review of the literature
}

\author{
Leroy ten Dam ${ }^{1} \cdot$ Diederik van de Beek ${ }^{1} \cdot$ Matthijs C. Brouwer ${ }^{1}$
}

Received: 7 September 2021 / Revised: 28 October 2021 / Accepted: 29 October 2021 / Published online: 7 November 2021

(c) The Author(s) 2021

\begin{abstract}
Hydrocephalus is reported in approximately one-tenth of neurosarcoidosis patients. However, data on clinical characteristics and outcome are lacking. In this retrospective study, we present 11 patients with neurosarcoidosis and hydrocephalus on neuroimaging. Median age was 52 years and seven were female (64\%). Presenting symptoms consisted of headache in 8 out of 11 (73\%), vertigo in 5 (46\%), gait abnormalities in 4 (36\%), diplopia in $2(18 \%)$ and decreased visual acuity in $1(9 \%)$. Cranial imaging showed obstructive hydrocephalus in $10(91 \%)$ and non-obstructive hydrocephalus in $1(9 \%)$ out of 11 , obstruction occurred at the level of the fourth ventricle in 6 out of $10(60 \%)$. Treatment consisted of glucocorticoids in all the patients with additional methotrexate or azathioprine in $6(55 \%)$ and infliximab in 1 (9\%) patient. Neurosurgical intervention was performed in 10 out of 11 (91\%) patients. Treatment led to remission, improvement or stabilization of disease in 9 out of 10 (90\%) of patients. One patient died due to cerebral herniation despite neurosurgical decompression and CSF shunting. Median modified Rankin scale score at last follow-up was 2 (range 0-6). A systematic review and meta-analysis of studies on hydrocephalus due to neurosarcoidosis identified 36 patients that compared to our patients had a lower median age at onset and a higher mortality. Acute obstructive hydrocephalus due to neurosarcoidosis is a potentially fatal medical emergency requiring neurosurgical intervention and initiation of immunosuppressive therapy. If patients survive the initial phase, the outcome is generally favorable.
\end{abstract}

Keywords Neurosarcoidosis $\cdot$ Auto-immune disease $\cdot$ Hydrocephalus $\cdot$ Cerebrospinal fluid

\section{Introduction}

Sarcoidosis is an inflammatory disorder that can involve multiple organ systems [1]. Involvement of the nervous system is called neurosarcoidosis and has been reported in $5-20 \%$ of patients with sarcoidosis. Neurosarcoidosis can affect all parts of both the central and peripheral nervous system [2]. The diagnosis of neurosarcoidosis depends on a combination of clinical characteristics and results of ancillary investigations mostly used to rule out other disorders [3]. Hydrocephalus due to neurosarcoidosis was found to occur in $9 \%$ of patients with neurosarcoidosis in a

Matthijs C. Brouwer

m.c.brouwer@amsterdamumc.nl

1 Department of Neurology, Amsterdam University Medical Center, University of Amsterdam, Amsterdam Neuroscience, Meibergdreef 9, 1105AZ Amsterdam, The Netherlands meta-analysis $[2,4,5]$. There are no large series describing clinical characteristics of patients with hydrocephalus due to neurosarcoidosis and data regarding imaging characteristics, optimal treatment strategy and outcome are lacking. In this retrospective single-center cohort study, we evaluated clinical features and treatment response of patients with hydrocephalus due to neurosarcoidosis. We also performed a systematic review and meta-analysis of the literature regarding hydrocephalus in neurosarcoidosis.

\section{Methods}

We retrospectively reviewed all case records from adult patients that were seen between June 2015 until June 2020 at the Neurology Department at the Amsterdam University Medical Centers a tertiary referral center for neurosarcoidosis. We included patients diagnosed with (1) neurosarcoidosis (either definite, probable or possible according to the 
criteria from the Neurosarcoidosis Consortium Consensus Group [3]) and (2) hydrocephalus on cranial imaging as reported by the neuroradiologist.

\section{Clinical data and ancillary investigations}

Detailed history and neurological examination were retrieved from patient files in an electronic database including presenting symptoms, age of onset of symptoms, results of ancillary investigations and data on treatment and treatment response and outcome of hydrocephalus in neurosarcoidosis.

\section{Review of the literature}

A literature search was performed in PubMed using the following terms: ("sarcoidosis" [MeSH Terms] OR sarcoid* [tiab] OR "Neurosarcoidosis" [Supplementary Concept] OR neurosarcoid* [tiab]) AND ("hydrocephalus" [MeSH Terms] OR hydrocephalus [tiab]). We included articles written in English describing one or more adult patients that were published in the last 25 years. The search was followed by a manual search in the reference lists of the publications found. The study conforms with the World Medical Association Declaration of Helsinki, 7th revision 2013 Fortaleza. Ethical approval is not required in the Netherlands for a retrospective study with anonymized patient data such as used in our study.

\section{Results}

Between June 2015 and June 2020, 303 patients were analyzed because of suspected neurological involvement of sarcoidosis at our outpatient department or clinical department. Of these, 153 patients (50\%) were diagnosed with neurosarcoidosis (either definite, probable or possible according to the criteria from the Neurosarcoidosis Consortium Consensus Group [3]). Hydrocephalus was present in 11 neurosarcoidosis patient (7\%; Table 1) of whom 7 were female (64\%). Median age at onset was 52 years (range 33-71 years).

Five patients (46\%) had a history of sarcoidosis of which one had a history of neurosarcoidosis-associated myelitis (9\%) and three used immunosuppressive therapy at symptom onset. Presenting symptoms were headache in eight (73\%), vertigo in five (46\%), abnormal gait in four (36\%), diplopia in two (18\%) and decreased visual acuity in one patient $(9 \%)$. Time from symptom onset of hydrocephalus-related symptoms to first neurological evaluation was 15 weeks (interquartile range 3-38). Abnormalities on neurological examination included nystagmus and impaired consciousness each in four (36\%), papilledema and ataxia each in two (18\%) and bilateral sixth nerve palsy in one patient $(9 \%)$. Neurological examination showed no abnormalities in two patients
(18\%). Median time from onset of symptoms to diagnosis of neurosarcoidosis was 4 months (range $0-34$ ). Other organ systems involved were lymph nodes in four patients (36\%), lungs in three (27\%) and eyes, liver and bones, respectively, in one patient.

Serum angiotensin-converting enzyme (ACE) was analyzed in five patients and was elevated in none of them, soluble Interleukin 2 receptor (sIL-2R) was evaluated in two patients and was elevated in both. Cerebrospinal fluid (CSF) analysis was performed, commonly after external ventricular drain placement, in nine and showed an elevated leukocyte count in eight (89\%) patients and elevated total protein in five (56\%). One patient had a normal CSF analysis. CSF oligoclonal bands were present in the one patient in which they were tested.

Results of cranial imaging were re-evaluated for all patients [ten magnetic resonance imaging (MRI) and one computed tomography (CT)] and showed hydrocephalus in all. The hydrocephalus was non-communicating/obstructive in ten patients and communicating in one. Obstruction was at the level of the fourth ventricle in six patients $(60 \%)$, the foramen of Monro in three (30\%) and the cerebral aqueduct in one (10\%). In one patient, there was tonsillar herniation due to the obstruction hydrocephalus (Fig. 1). Post-contrast series were available in nine patients and showed abnormal leptomeningeal contrast enhancement in seven (78\%). Abnormal contrast enhancement was located at the level of the fourth ventricle (foramina of Magendie and Luschka, surrounding the medulla oblongata or vermis of the cerebellum) in six patients (Fig. 1). Other abnormalities were a contrast enhancing multicystic process near the foramen of Monro, enhancing punctiform nodules in the brain parenchyma and dural contrast enhancement, which were all observed in one patient.

Fluor-18-deoxyglucose positron emission tomography $\left({ }^{18}\right.$ FDG-PET) was performed in seven patients with no history of sarcoidosis, and showed abnormalities suggestive of sarcoidosis in all of them. Biopsy was performed in all patients. Leptomeningeal biopsy was performed in one patient and showed non-caseating granulomas (definite neurosarcoidosis), lymph node biopsy was performed in eight patients and showed non-caseating granulomas in seven (probable neurosarcoidosis) and was inconclusive in one (possible neurosarcoidosis), in one patient liver biopsy was performed and showed non-caseating granulomas (probable neurosarcoidosis). In the patient with possible neurosarcoidosis, chest CT was highly suggestive of sarcoidosis.

The median follow-up was 23.5 months (interquartile range 3-50 months). Immunosuppressive treatment was initiated in all 11 patients and consisted of corticosteroid pulse therapy in 7 (64\%), corticosteroid maintenance therapy in $9(82 \%)$ and second-line therapy, methotrexate or azathioprine, in 4 patients $(36 \%)$. Neurosurgical 
Table 1 Clinical characteristics, results of ancillary investigations, treatment and outcome in hydrocephalus due to neurosarcoidosis

\begin{tabular}{|c|c|c|c|}
\hline Characteristics & $n / N(\%)$ & Characteristics & $n / N(\%)$ \\
\hline Median age at onset (range) & \multicolumn{2}{|c|}{52 (33-71) Biopsy } & \\
\hline Sex (female) & $7 / 11(64 \%)$ & Non-caseating granulomas & $10 / 11(91 \%)$ \\
\hline History of sarcoidosis & $5 / 11(46 \%)$ & \multicolumn{2}{|l|}{ Classification of neurosarcoidosis [3] } \\
\hline Median weeks to evaluation (IQR) & $15(3-38)$ & Definite & $1 / 11(9 \%)$ \\
\hline Clinical symptoms at onset & & Probable & $9 / 11(82 \%)$ \\
\hline Headache & $8 / 11(73 \%)$ & Possible & $1 / 11(9 \%)$ \\
\hline Vertigo & $5 / 11(46 \%)$ & Immunosuppressive treatment & $11(100 \%)$ \\
\hline Gait abnormalities & $4 / 11(36 \%)$ & Corticosteroid pulse & $7(64 \%)$ \\
\hline Diplopia & 2/11 (18\%) & Corticosteroid maintenance & $9(82 \%)$ \\
\hline Decreased visual acuity & $1 / 11(9 \%)$ & Second-line therapy & $4(36 \%)$ \\
\hline Abnormalities at first evaluation & & Medication changes & $5 / 11(45 \%)$ \\
\hline Impaired consciousness & $4 / 11(36 \%)$ & Corticosteroid therapy & $3 / 5(60 \%)$ \\
\hline Papilledema & $2 / 11(18 \%)$ & Second-line therapy & $5 / 5(100 \%)$ \\
\hline Nystagmus & $4 / 11(36 \%)$ & Third-line therapy & $1 / 5(20 \%)$ \\
\hline Cranial nerve palsy & $1 / 11(9 \%)$ & Neurosurgical intervention & $10 / 11(91 \%)$ \\
\hline Ataxia & $2 / 11(18 \%)$ & CSF shunt & $8 / 10(80 \%)$ \\
\hline Sarcoidosis localisation & & Ventriculostomy & $3 / 10(30 \%)$ \\
\hline Lung & $3 / 11(27 \%)$ & Cyst fenestration & $1 / 10(10 \%)$ \\
\hline Lymph nodes & $4 / 11(36 \%)$ & Follow-up & \\
\hline Other & $3 / 11(27 \%)$ & Median duration in months (IQR) & $23.5(0-50)$ \\
\hline Laboratory investigations & & Median mRS score (range) & $2(0-6)$ \\
\hline Elevated ACE & $0 / 5(0 \%)$ & Outcome of neurosarcoidosis & \\
\hline Elevated sIL-2R & $2 / 2(100 \%)$ & Remission & $2 / 11(18 \%)$ \\
\hline Elevated CSF leukocyte count & $8 / 9(89 \%)$ & Improvement & $5 / 11(45 \%)$ \\
\hline Elevated CSF total protein & $5 / 9(56 \%)$ & Stable disease & $3 / 11(27 \%)$ \\
\hline Cranial imaging & & Death & $1 / 11(9 \%)$ \\
\hline Communicating hydrocephalus & $1 / 11(9 \%)$ & & \\
\hline Obstructive hydrocephalus & $10 / 11(91 \%)$ & & \\
\hline Obstruction at 4th ventricle & $6 / 10(60 \%)$ & & \\
\hline Obstruction at foramen of Monro & $3 / 10(30 \%)$ & & \\
\hline Obstruction at cerebral aqueduct & $1 / 10(10 \%)$ & & \\
\hline Meningeal enhancement & $7 / 9(78 \%)$ & & \\
\hline
\end{tabular}

The upper limit of normal of normal (ULN) of ACE is $40 \mathrm{U} / \mathrm{L}$ and the ULN of sIL-2R is $555 \mathrm{U} / \mathrm{L}$. The ULN of cerebrospinal fluid analysis for leukocytes is $4 \times 10^{6} / \mathrm{L}$ and for protein is $0.6 \mathrm{~g} / \mathrm{L}$

$A C E$ angiotensin-converting enzyme, CSF cerebrospinal fluid, $I Q R$ interquartile range, $m R S$ modified Rankin scale, $s I L 2-R$ soluble interleukin 2 receptor intervention was performed in 10 out of 11 patients $(91 \%)$ and consisted of external ventricular drain placement followed by ventriculoperitoneal shunt in 3 (30\%), ventriculoperitoneal shunt in $5(50 \%)$, ventriculostomy in $3(30 \%)$ and cyst fenestration in 1 patient (10\%). All but one patient were treated both with immunosuppressive agents as well as a neurosurgical intervention. Of these ten patients, three underwent neurosurgical intervention prior to immunosuppressive treatment, three received immunosuppressive treatment prior to neurosurgery and in four, neurosurgical and immunosuppressive treatments were initiated simultaneously. Medication was changed during follow-up due to insufficient response to initial therapy, side effects or a relapse of symptoms during follow-up in 5 out of 11 patients (45\%). One patient had multiple relapses of symptoms. The relapse of symptoms of neurosarcoidosis was recurrence of hydrocephalus in four $(80 \%)$ and meningitis without hydrocephalus and myelitis each in one patient (20\%). Treatment changes included restarting or increasing the dosage of corticosteroid maintenance therapy in three $(80 \%)$, corticosteroid pulse therapy in three $(60 \%)$ and initiation of second-line therapy in five ( $80 \%$; methotrexate in three and azathioprine in two). Third-line therapy (infliximab) was started in one patient (20\%). Two patients underwent a second neurosurgical intervention because of failure of CSF shunt or ventriculostomy. 
Fig. 1 Obstructive hydrocephalus in neurosarcoidosis. Mid-sagittal contrast enhanced T1-weighted image (A) showing dilatation of the third and fourth ventricles and leptomeningeal enhancement of the bottom of the fourth ventricle. Axial CT scan (B) showing quadriventricular hydrocephalus and tonsillar herniation. Midsagittal T2-SPACE $(\mathbf{C})$ showing a trapped fourth ventricle. Axial T2-FLAIR (D) showing dilatation of the right occipital horn due to a multicystic process located near the foramen of Monro
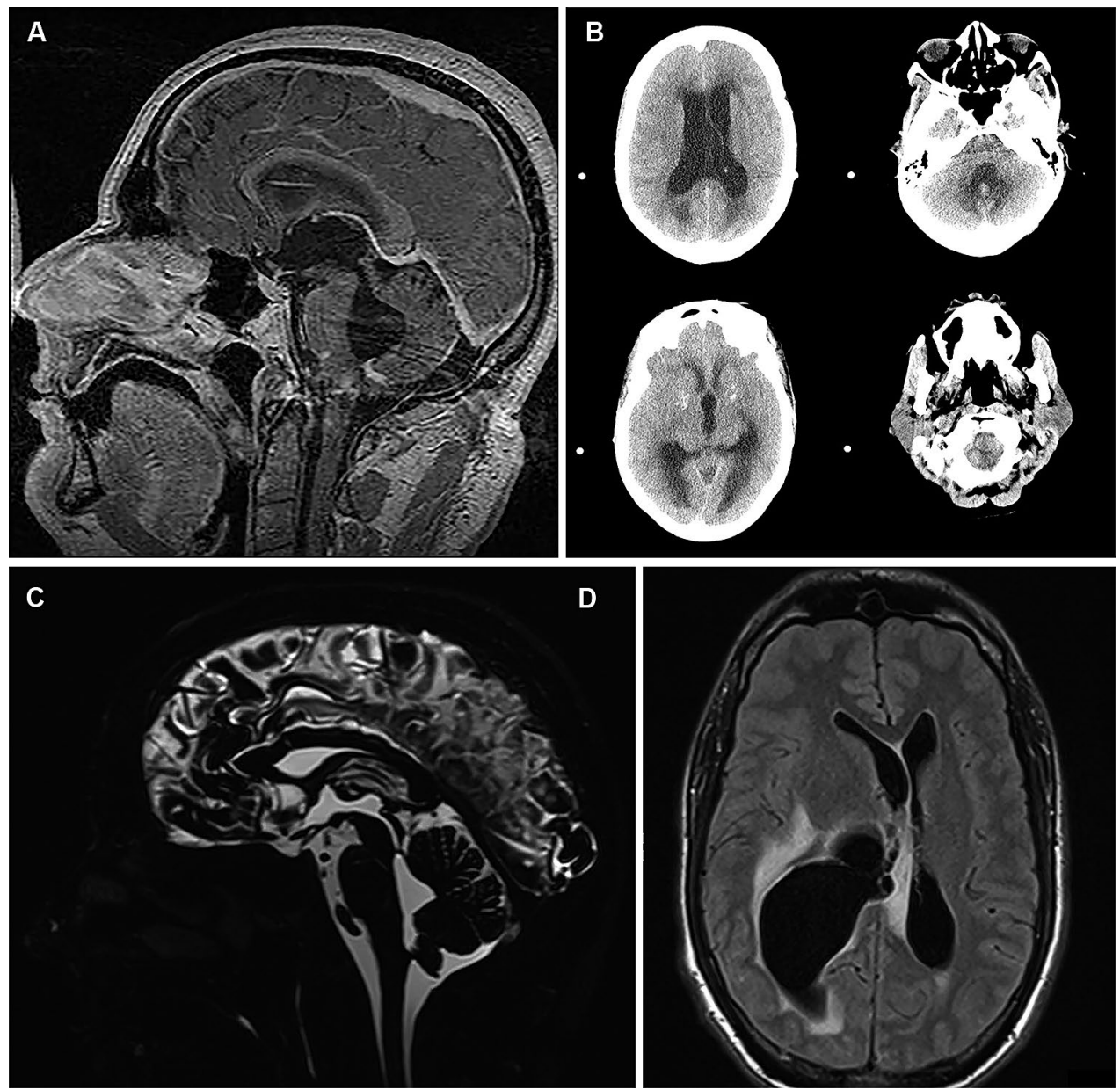

Neurological examination at the end of follow-up was available in ten patients and focal neurological deficits were present in only one patient and were attributable to sarcoidosis-associated myelitis. Outcome of neurosarcoidosis at last follow-up was classified as remission in two (18\%), improvement in five $(45 \%)$ and stable disease in three $(27 \%)$. One patient (9\%) died following cerebral herniation as a result of the hydrocephalus. Median modified Rankin scale (mRS) score at last follow-up was 2 (range 0-6).

\section{Literature review}

Literature review yielded 95 articles of which 46 were assessed for eligibility based on title and abstract. Of these articles, five consisted of case series of neurosarcoidosis patients that included patients with hydrocephalus but did not report clinical characteristic, ancillary investigations, treatment and outcome of this subgroup [4, 6-9]. Seven articles were excluded based on language and one article reported on a patient that is included in our case series. Thirty-three articles describing 36 patients met our inclusion criteria (Table 2) [10-42]. The median age was 32 years (range 17-67) and 17 out of 35 patients were female (49\%).
Symptoms at onset were headache in $20(56 \%)$, impaired consciousness in 16 (44\%), nausea or vomiting in $14(39 \%)$ and visual disturbances in $9(25 \%)$ out of 36 patients. Elevated CSF leukocyte count was reported in 16 out of $22(73 \%)$ and elevated total protein in 13 out of $21(62 \%)$ patients. Cranial imaging showed hydrocephalus in all patients. The hydrocephalus was communicating in $9(38 \%)$ and obstructive in 15 out of 24 (63\%) of patients. Obstruction was at the level of the fourth ventricle in seven $(47 \%)$, the cerebral aqueduct in five $(33 \%)$ or above the level of the cerebral aqueduct in three patients $(20 \%)$. Meningeal contrast enhancement was seen in 20 out of 25 patients $(80 \%)$. Neurosarcoidosis was classified as definite in $20(57 \%)$ and as probable in 15 (43\%) out of 35 patients. Most patients were treated with steroids [31 out of 35 (89\%)]. Secondline treatment consisted of azathioprine and methotrexate both in 4 of 35 (11\%) patients. Third-line treatment with infliximab was started in two $(6 \%)$ patients. Four out of $35(11 \%)$ patients were started on other immunosuppressive agents which included mycophenolate mofetil, cyclophosphamide and cyclosporine. Neurosurgical intervention was performed in 29 out of $36(81 \%)$ patients and included permanent CSF shunts in $21(72 \%)$, ventriculostomy in 6 
Table 2 Clinical characteristics, ancillary investigations, treatment and outcome in patients with hydrocephalus due to neurosarcoidosis from the literature

\begin{tabular}{lclc}
\hline Characteristics & $n / N(\%)$ & Characteristics & $n / N(\%)$ \\
\hline Median age at onset (range) & $32(17-67)$ & Classification of neurosarcoidosis & \\
Sex (female) & $17 / 35(49 \%)$ & Definite & $20 / 35(57 \%)$ \\
Clinical symptoms at onset & & Probable & $15 / 35(43 \%)$ \\
Headache & $20 / 36(56 \%)$ & Possible & $0 / 35(0 \%)$ \\
Visual disturbances & $9 / 36(25 \%)$ & Cumulative treatment \\
Nausea or vomiting & $14 / 36(39 \%)$ & Corticosteroid therapy & $31 / 35(89 \%)$ \\
Impaired consciousness & $16 / 36(44 \%)$ & Methotrexate & $4 / 35(11 \%)$ \\
Laboratory investigations & & Azathioprine & $4 / 35(1 \%)$ \\
Elevated CSF leukocyte count & $16 / 22(73 \%)$ & Infliximab & $2 / 35(6 \%)$ \\
Elevated CSF total protein & $13 / 21(62 \%)$ & Other immunosuppressants & $4 / 35(11 \%)$ \\
Cranial imaging & & Neurosurgical intervention & $29 / 36(81 \%)$ \\
Communicating hydrocephalus & $9 / 24(38 \%)$ & CSF shunt & $21 / 30(70 \%)$ \\
Obstructive hydrocephalus & $15 / 24(63 \%)$ & Ventriculostomy & $6 / 30(20 \%)$ \\
Obstruction at 4th ventricle & $7 / 15(47 \%)$ & Other neurosurgical intervention & $9 / 30(30 \%)$ \\
Obstruction at cerebral aqueduct & $5 / 15(33 \%)$ & Relapse of symptoms & $8 / 28(29 \%)$ \\
Other site of obstruction & $3 / 15(20 \%)$ & Outcome of neurosarcoidosis & \\
Meningeal enhancement & $20 / 25(80 \%)$ & Improvement & $27 / 36(75 \%)$ \\
Enhancement at 4th ventricle & $8 / 20(40 \%)$ & Deterioration & $1 / 36(3 \%)$ \\
Enhancement at cerebral aqueduct & $3 / 20(15 \%)$ & Death & $8 / 36(22 \%)$ \\
Other site of contrast enhancement & $15 / 20(75 \%)$ & & \\
\hline
\end{tabular}

CSF cerebrospinal fluid
(21\%) and other interventions such as endoscopic fenestration, partial lobectomy, open brain biopsy and temporary external CSF shunt in 9. Outcome of neurosarcoidosis was described as improvement in $27(75 \%)$ patients, deterioration in $1(3 \%)$ and deterioration leading to death in 8 out of 36 patients (22\%). Four patients died due to cerebral herniation. In the other patients, cause of death was intractable seizures, treatment-related complications and pulmonary embolism.

\section{Discussion}

Our study shows that hydrocephalus is a rare but potentially fatal manifestation of neurosarcoidosis that requires a combination of neurosurgical intervention and immunosuppressive treatment. In the absence of a positive history of (neuro)sarcoidosis, the differential diagnosis of acquired hydrocephalus is diverse and includes among others inflammatory, (post-) infectious, (post-)hemorrhagic and (post-) traumatic disorders and intracranial tumors such as colloid cysts or malignancy $[5,12,43,44]$. To our knowledge, there is no literature regarding the percentage of hydrocephalus cases caused by neurosarcoidosis. As hydrocephalus was often the first manifestation of neurosarcoidosis ancillary investigations in acquired hydrocephalus with an unknown etiology should include sarcoidosis biomarkers (e.g., ACE and s-IL2R) and a chest CT and if normal ${ }^{18}$ FDG-PET. These investigations can help ascertain a potential biopsy site outside of the central nervous system. Histopathological evidence of non-caseating granulomas is necessary for a diagnosis of probable or definite neurosarcoidosis [3, 45].

Presenting symptoms of hydrocephalus in neurosarcoidosis are (sub)acute and related to increased intracranial pressure. Hydrocephalus in neurosarcoidosis is often noncommunicating and caused by obstruction of the outflow from the fourth ventricle or of the cerebral aqueduct due to a chronic meningitis. Acute obstructive hydrocephalus can be fatal and an emergency neurosurgical intervention such as CSF shunting, ventriculostomy or decompression should be considered in all patients. Corticosteroid treatment in adjunct to neurosurgical intervention should be strongly considered in all patients. Intensification of immunosuppressive treatment is required in case of a relapse of symptoms, as a persistent or recurring granulomatous chronic meningitis can lead to an additional site of obstruction or shunt failure through obstruction of the CSF shunt [41]. Although relapses of symptoms and medication switches were frequent, there was stabilization, improvement or remission of neurosarcoidosis in nearly all patients that survived the initial phase.

As compared to our case series, the mortality was higher (9\% versus $22 \%$ ) and more patients had a diagnosis of definite neurosarcoidosis ( $10 \%$ versus $58 \%$ ) in the review of the literature. The difference in mortality may be explained by publication bias as multiple articles reported on patients with sudden death. A previous review of the 
literature found an even higher mortality of $75 \%$ in patients with neurosarcoidosis who presented with seizures and had hydrocephalus [46]. However, mortality in this group was high (47\%) irrespective of hydrocephalus. The higher percentage of patient with definite neurosarcoidosis was due to CNS biopsy being performed more often alongside neurosurgical treatment of the acute hydrocephalus in cases reported in the literature.

This study has several limitations. First, patients evaluated were referred to our tertiary center. This may introduce selection bias leading to overestimation of the impact of hydrocephalus in neurosarcoidosis. Second, the retrospective design of our study resulted in heterogeneous assessment of disease activity as well as missing data in some patients. This prohibits drawing firm conclusions regarding outcome and treatment effect. Third, as mentioned above there might be publication bias regarding hydrocephalus in neurosarcoidosis. Nevertheless, our retrospective single-center cohort study and systematic review provides valuable information on a rare disease as hydrocephalus in neurosarcoidosis.

In conclusion, hydrocephalus due to neurosarcoidosis is a medical emergency as onset is often (sub)acute and the hydrocephalus is obstructive in nature. Initiation of treatment should be prompt and include both neurosurgical interventions to lower intracranial pressure and immunosuppressive therapy to reduce granulomatous inflammation. If patients survive the initial phase outcome is generally favorable.

Acknowledgements DvdB is supported by a grant from the Netherlands Organization for Health Research and Development (ZonMw; NWO-Vici Grant 2019 [918.19.627]) and an Innovation grant by the board of directors of the Amsterdam UMC, Amsterdam, the Netherlands. MCB is supported by grants from the Netherlands Organization for Health Research and Development (ZonMw; NWO-Vidi Grant 2017 [917.17.308]) and the European Research Council (ERC Consolidator Grant 101001237). No potential conflict of interest relevant for this article exist.

Author contributions All the authors contributed to the study conception and design. Material preparation, data collection and analysis were performed by LD. The first draft of the manuscript was written by LD and all the authors commented on previous versions of the manuscript. All the authors read and approved the final manuscript.

Funding No funding was received for conducting this study.

Data availability The data that support the findings of this study are available from the corresponding author upon request.

Code availability Not applicable.

\section{Declarations}

Conflicts of interest The authors declare that they have no conflict of interest.
Ethics approval The study conforms with the World Medical Association Declaration of Helsinki, 7th revision 2013 Fortaleza. Ethical approval is not required in the Netherlands for a retrospective study with anonymized patient data such as used in our study.

Open Access This article is licensed under a Creative Commons Attribution 4.0 International License, which permits use, sharing, adaptation, distribution and reproduction in any medium or format, as long as you give appropriate credit to the original author(s) and the source, provide a link to the Creative Commons licence, and indicate if changes were made. The images or other third party material in this article are included in the article's Creative Commons licence, unless indicated otherwise in a credit line to the material. If material is not included in the article's Creative Commons licence and your intended use is not permitted by statutory regulation or exceeds the permitted use, you will need to obtain permission directly from the copyright holder. To view a copy of this licence, visit http://creativecommons.org/licenses/by/4.0/.

\section{References}

1. Valeyre D, Prasse A, Nunes H, Uzunhan Y, Brillet PY, MüllerQuernheim J (2014) Sarcoidosis. Lancet 383:1155-1167

2. Fritz D, Van De Beek D, Brouwer MC (2016) Clinical features, treatment and outcome in neurosarcoidosis: systematic review and meta-analysis. BMC Neurol 16:220

3. Stern BJ, Royal W 3rd, Gelfland JM, Clifford DB, Tavee J, Pawate S, Berger JR, Aksamit AJ, Krumholz A, Pardo CA, Moller DR, Judson MA, Drent M, Baughman RP (2018) Definition and consensus diagnostic criteria for neurosarcoidosis: from the Neurosarcoidosis Consortium Consensus Group. JAMA Neurol 75:1546-1553

4. Bathla G, Freeman CW, Moritani T, Song JW, Srivastava S, Soni N, Derdeyn C, Mohan S (2020) Retrospective, dual-centre review of imaging findings in neurosarcoidosis at presentation: prevalence and imaging sub-types. Clin Radiol 75(796):E791-796.E799

5. Wei J, Yin H, Wang L, Cui L, Wang R (2019) Systemic autoimmune diseases complicated with hydrocephalus: pathogenesis and management. Neurosurg Rev 42:255-261

6. Goel J, Anadure R, Gupta S, Wilson V, Saxena R, Sahu S, Mutreja D (2020) A study of the clinical profile, radiologic features, and therapeutic outcomes in neurosarcoidosis from two tertiary care centers in southern India. Neurol India 68:609-616

7. Blume C, Tuleta I, Nolte K, Eichhorn KW, Jakob M, Clusmann H, Send T (2020) Neurosarcoidosis as a rare differential diagnosis for single or multiple lesions of the nervous system. Br J Neurosurg 34:495-499

8. Kidd DP (2018) Sarcoidosis of the central nervous system: clinical features, imaging, and CSF results. J Neurol 265:1906-1915

9. Allen RK, Sellars RE, Sandstrom PA (2003) A prospective study of 32 patients with neurosarcoidosis. Sarcoidosis Vasc Diffuse Lung Dis 20:118-125

10. Alfraji N, Douedi S, Hosseini MA (2020) Hydrocephalus and myelopathy: a rare and challenging case of sarcoidosis and review of literature. Clin Case Rep 8:1890-1894

11. Togashi S, Maruya J, Abe H, Nishimaki K, Ouchi H, Hara K, Tokairin T, Nishiyama K, Shimizu H (2020) Endoscopic management for recurrent hydrocephalus associated with neurosarcoidosis. World Neurosurg 144:121-124

12. Saban RJ, Berns MM, Al-Hakim MM, Patino GA (2020) Hydrocephalus as the presenting symptom of sarcoidosis: a case report and review of literature. Clin Case Rep 8:363-368 
13. Mckeever A, Cox A, Garnett M, Cuniffe NG (2019) Hydrocephalus as the first presenting symptom of neurosarcoidosis in two patients: a diagnosis more forthcoming in the context of systemic disease. BMJ Case Rep 12:e229903

14. Nakayasu H, Shirai T, Tanaka Y, Sato T, Muro H (2019) Neurosarcoidosis presenting with obstructive hydrocephalus successfully treated with endoscopic third ventriculostomy. Intern Med 58:105-108

15. Wang F, Guo D, Liu Z, Zhou A, Wei C, Jia J (2018) Neurosarcoidosis: clinical characteristics, diagnosis, and treatment in eight Chinese patients. Neurol Sci 39:1725-1733

16. Labarca G, Ramirez R, Monsalve X, Mira-Avendano I (2016) Dementia, gait disturbance, and urinary incontinence in a patient with pulmonary sarcoidosis. Respirol Case Rep 4:E00182

17. Radwan W, Lucke-Wold B, Robadi IA, Gyure K, Roberts T, Bhatia S (2017) Neurosarcoidosis: unusual presentations and considerations for diagnosis and management. Postgrad Med J 93:401-405

18. Sano H, Deguchi I, Fukuoka T, Hayashi T, Uchino A, Adachi J, Yasuda M, Takao M, Tanahashi N (2016) Intractable neurosarcoidosis effectively treated with infliximab. Intern Med $55: 811-814$

19. Yoshitomi M, Uchikado H, Hattori G, Sugita Y, Morioka M (2015) Endoscopic biopsy for the diagnosis of neurosarcoidosis at the fourth ventricle outlet with hydrocephalus. Surg Neurol Int 6:S633-636

20. Kurata I, Tsuboi H, Takahashi H, Yagishita M, Abe S, Ebe H, Takahashi H, Asashima H, Hirota T, Hagiwara S, Umeda N, Kondo Y, Ogishima H, Suzuki T, Matsumoto I, Sumida T (2015) A Case of relapsing neurosarcoidosis with brain nodules and hydrocephalus successfully treated by corticosteroid and methotrexate. Rheumatology (Oxford) 54:1160

21. Chandna A, Todd C, Murphy D, Boet R, Duncan R (2015) Sarcoidosis presenting with acute hydrocephalus in a New Zealand European female. N Z Med J 128:110-113

22. Tabuchi S, Uno T (2013) Hydrocephalus with panventricular enlargement as the primary manifestation of neurosarcoidosis: a case report. J Med Case Rep 7:240

23. Zoja R, Andreola S, Gentile G, Rancati A (2012) Sudden death from systemic sarcoidosis: a case of legal medicine. Sarcoidosis Vasc Diffuse Lung Dis 29:62-68

24. Kim SH, Lee SW, Sung SK, Son DW (2012) Treatment of hydrocephalus associated with neurosarcoidosis by multiple shunt placement. J Korean Neurosurg Soc 52:270-272

25. van Rooijen JM, Mijnhout GS, Aalders TT, de Bondt RB (2011) Hydrocephalus, a rare manifestation of sarcoidosis. Clin Pract 1:E66

26. Lee Ch, Jung YS, Lee Sh (2010) Hydrocephalus as a presenting manifestation of neurosarcoidosis: easy to misdiagnose as tuberculosis. J Korean Neurosurg Soc 48:79-81

27. Berhouma M, Abderrazek K, Krichen W, Jemel H (2009) Apropos of an unusual and menacing presentation of neurosarcoidosis: the space-occupying trapped temporal horn. Clin Neurol Neurosurg 111:196-199

28. Westhout FD, Linskey ME (2008) Obstructive hydrocephalus and progressive psychosis: rare presentations of neurosarcoidosis. Surg Neurol 69:288-292 (Discussion 292)
29. Benzagmout M, Boujraf S, Góngora-Rivera F, Bresson D, Van-Effenterre R (2007) Neurosarcoidosis which manifested as acute hydrocephalus: diagnosis and treatment. Intern Med 46:1601-1604

30. Miscusi M, Polli FM, Missori P, Delfini R (2006) Ghost lesions in patient with cerebral-isolated neurosarcoidosis. A case report. J Neurosurg Sci 50:17-20 (Discussion 20)

31. Muniesa C, Marcoval J, Moreno A, Mañá J, Ferreres JR, Peyrí J (2006) Hydrocephalic neurosarcoidosis diagnosed by cutaneous lesions. J Am Acad Dermatol 55:S125-126

32. Muayqil T, Ms H, Saqqur M (2006) A patient with neurosarcoidosis. Can J Neurol Sci 33:92-94

33. Hamada H, Hayashi N, Kurimoto M, Umemura K, Hirashima $\mathrm{Y}$, Endo S (2004) Isolated third and fourth ventricles associated with neurosarcoidosis successfully treated by neuroendoscopy-case report. Neurol Med Chir (Tokyo) 44:435-437

34. Akhondi H, Barochia S, Holmström B, Williams MJ (2003) Hydrocephalus as a presenting manifestation of neurosarcoidosis. South Med J 96:403-406

35. Hesselmann V, Wedekind C, Terstegge K, Schulte O, Voges J, Krug B, Lackner K (2002) An isolated fourth ventricle in neurosarcoidosis: MRI findings. Eur Radiol 12(Suppl 3):S1-S3

36. Scott TF, Brillman J (2000) Shunt-responsive dementia in sarcoid meningitis: role of magnetic resonance imaging and cisternography. J Neuroimaging 10:185-186

37. Nakamura A, Ohara S, Maruyama K, Takei Y, Shindo M, Yanagisawa N (1999) Systemic sarcoidosis: a case with a focal hydrocephalus and elevated lysozyme and angiotensin-converting enzyme in the cerebrospinal fluid. J Neurol 246:320-322

38. Thomas G, Murphy S, Staunton H, Oneill S, Farrell M, Brett F (1998) Pathogen-free granulomatous diseases of the central nervous system. Hum Pathol 29:110-115

39. Sattelmeyer VM, Vernet O, Janzer R, De Tribolet N (1999) Neurosarcoidosis presenting as an isolated mass of the quadrigeminal plate. J Clin Neurosci 6:259-261

40. Sugita M, Sano M, Uchigata M, Aruga T, Matsuoka R (1997) Facial nerve enhancement on gadolinium-DTPA in a case with neurosarcoidosis. Intern Med 36:825-828

41. Maniker Ah, Cho ES, Schulder M (1997) Neurosarcoid infiltration of the ventricular catheter causing shunt failure: a case report. Surg Neurol 48:527-529

42. Maisel AJ, Lynam T (1996) Unexpected sudden death in a young pregnant woman: unusual presentation of neurosarcoidosis. Ann Emerg Med 28:94-97

43. Shemie S, Jay V, Rutka J, Armstrong D (1997) Acute obstructive hydrocephalus and sudden death in children. Ann Emerg Med 29:524-528

44. Maller VV, Gray RI (2016) Noncommunicating hydrocephalus. Semin Ultrasound CT MRI 37:109-119

45. Fritz D, van de Beek D, Brouwer MC, Booij J (2020) Whole-body ${ }^{18}$ F-FDG PET-CT in the diagnosis of neurosarcoidosis. Mayo Clin Proc 95:1082-1084

46. Sponsler JL, Werz MA, Maciunas R, Cohen M (2005) Neurosarcoidosis presenting with simple partial seizures and solitary enhancing mass: case reports and review of the literature. Epilepsy Behav 6:623-630 